\title{
An Unusual Case of Multiple Primary Malignancies with Fungal Infection
}

\author{
${ }^{1}$ KN Mohan Rao, ${ }^{2}$ Gundappa Vivek, ${ }^{3}$ SV Siva P Reddy
}

\begin{abstract}
A 50-year-old male presented with complaints of cough with expectoration since 2 months with pain during defecation and loose stools since 1 month, and one episode of hemoptysis. Chest X-ray showed bilateral multiple nodular opacities with multiple cavities with air fluid levels. Sputum acid-fast bacilli (AFB) fungal culture showed growth of Aspergillus terreus. Computed tomography (CT) scan of chest and abdomen showed bilateral multiple cavities and nodules with left pleural effusion and adrenal metastasis with rectal mass and narrowing of lumen. Rectal biopsy showed moderately differentiated adenocarcinoma. Computed tomography-guided lung biopsy was also done, which surprisingly came as poorly differentiated large cell carcinoma.

We report a case of synchronous multiple primary malignancy (MPM), rectum and lung, primary lung metastasizing to adrenal glands and lung with secondary fungal infection (A. terreus).
\end{abstract}

Keywords: Multiple primary malignancies, Multiple primary malignancy with fungal infection, Rectal carcinoma.

How to cite this article: Rao KNM, Vivek G, Reddy SVSP. An Unusual Case of Multiple Primary Malignancies with Fungal Infection. J Med Sci 2017;3(3):86-88.

Source of support: Nil

Conflict of interest: None

\section{INTRODUCTION}

Multiple primary malignancies in a single host have seen an increasing trend during the past decade. ${ }^{1}$ The term "multiple primary malignancy" was first used by Billroth in 1889, and the first paper describing MPM was published by Warren and Gates in $1932 .{ }^{2}$ Multiple primary malignancies are defined as two or more independent primary malignancies of different histologies/ origins in the same individual. They are divided into two categories depending on the time of diagnosis of each tumor. If the tumors are diagnosed simultaneously or within a 6-month interval, they are called synchronous.

\footnotetext{
${ }^{1}$ Professor and Head, ${ }^{2,3}$ Postgraduate Student

${ }^{1-3}$ Department of Pulmonary Medicine, RajaRajeswari Medical College \& Hospital, Bengaluru, Karnataka, India

Corresponding Author: Gundappa Vivek, Postgraduate Student Department of Pulmonary Medicine, RajaRajeswari Medical College \& Hospital, Bengaluru, Karnataka, India, e-mail: kotnur. rao@gmail.com
}

If the interval is longer than 6 months, the tumors are called metachronous. ${ }^{2}$

The risk is higher in women for metachronous tumors, but synchronous lesions usually favor men. ${ }^{3}$ Despite its increasing rates, MPMs are rare with an incidence between 0.4 and $18.4 \%$ in various countries and various studies. $^{2}$

Because of the poor awareness of the condition, some patients who suffer from MPM may be misdiagnosed with metastatic carcinoma. It should be clearly differentiated from primary malignancies and metastasis, so that treatment can be directed accordingly.

The incidence of Aspergillus infections among cancer patients has increased over the recent past, despite overall advances in supportive care. ${ }^{4}$ Here, we report a rare case of synchronous MPM with secondary fungal infection.

\section{CASE REPORT}

A 50-year-old male farmer presented with complaints of cough with expectoration since 2 months associated with left-sided chest pain for the past 15 days. He also had loose stools since the past 1 month, four to five times per day, associated with pain during defecation. He had an episode of hemoptysis about $50 \mathrm{~mL}$ frank blood prior to presentation to the hospital and complained of loss of appetite and loss of weight. He is a smoker with 40 pack years and nonalcoholic. He is not a known diabetic or hypertensive.

On examination, patient was anemic, with grade III clubbing and had no lymphadenopathy. Respiratory and cardiac examination was normal. Per rectal examination revealed a hard growth in the rectum (Fig. 1).

Chest X-ray showed bilateral multiple nodular opacities with air fluid levels. Hemoglobin was $9.4 \mathrm{gm} / \mathrm{dL}$, Erythrocyte sedimentation rate was $40 \mathrm{~mm}$ at the end of 1 hour, total counts were 7,600 cells/cu mm. Renal and liver parameters were normal. Sputum AFB and Gram stain and human immunodeficiency virus were negative. Potassium hydroxide mount and fungal culture showed growth of A. terreus. Serum immunoglobulin E levels were $1,239 \mathrm{IU} / \mathrm{mL}$.

Biopsy was taken from the rectal growth, which showed moderately differentiated adenocarcinoma. The $\mathrm{CT}$ scan of chest and abdomen was done which showed bilateral multiple cavities and nodules with minimal left 


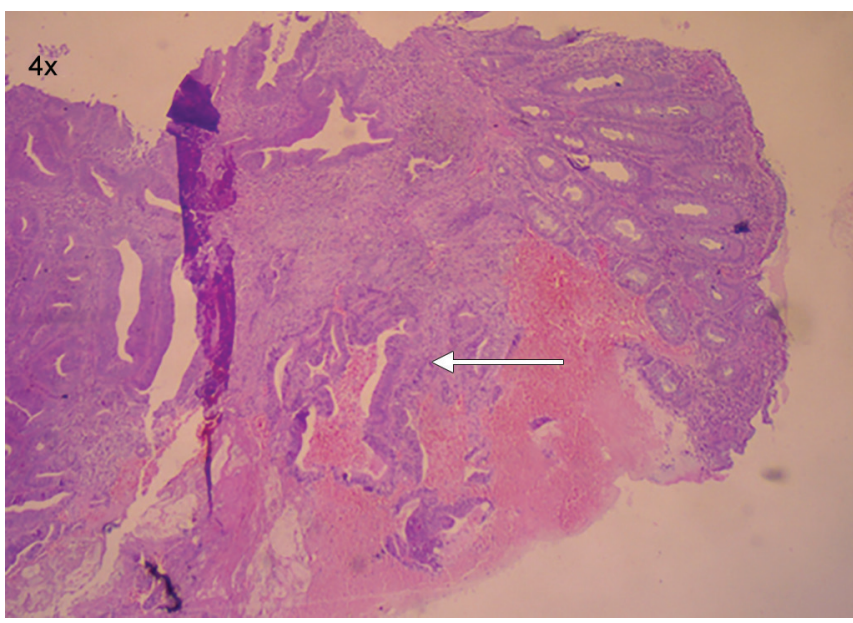

Fig. 1: Normal mucosa with infiltrating tumor cells in glandular pattern seen

pleural effusion and adrenal metastasis with circumferential rectal wall thickening and mass causing narrowing of the lumen. Later transthoracic lung biopsy was done, which surprisingly showed poorly differentiated large cell carcinoma (Fig. 2). Patient was started on chemotherapy and voriconazole for fungal infection.

\section{DISCUSSION}

With increase in number of cancer patients, the risk of developing multiple primary malignant tumors is also increasing. The etiology is still unclear, but multiple mechanisms have been implicated in the pathogenesis, like hereditary factors, environmental factors, such as chemicals, asbestos exposure, smoking, chemotherapeutic regimens, viruses, and ionizing radiation. ${ }^{1}$

Furthermore, a number of hereditary conditions are associated with multiple primary malignant neoplasms. Li-Fraumeni syndrome, which is a rare disorder, is associated with CHEK2 and TP53 genes. Various genetic changes, such as punctiform mutations, loss of heterozygosity, or genetic instability, microsatellite instability, occur more frequently and have a worse prognosis in cases of MPMs than sporadic cancers. ${ }^{5,6}$

Schoenberg ${ }^{7}$ found that patients with cancer had 1.29 times higher risk of developing a new malignancy compared with those who were never diagnosed. Goya et $\mathrm{al}^{8}$ reported that lung metastases develop in 10 to $20 \%$ of patients with colorectal carcinoma. ${ }^{9}$ But in some rare cases, patients can develop synchronous colorectal and lung cancer.

Evans et al investigated 1,27,281 patients with colorectal cancer and found that 801 cases $(0.6 \%)$ had also developed primary lung malignancy, while the incidence of synchronous colorectal and lung cancer is much lower in these patients. ${ }^{10}$

For the accurate classification of MPM, each tumor must exhibit a definite picture of malignancy, each tumor

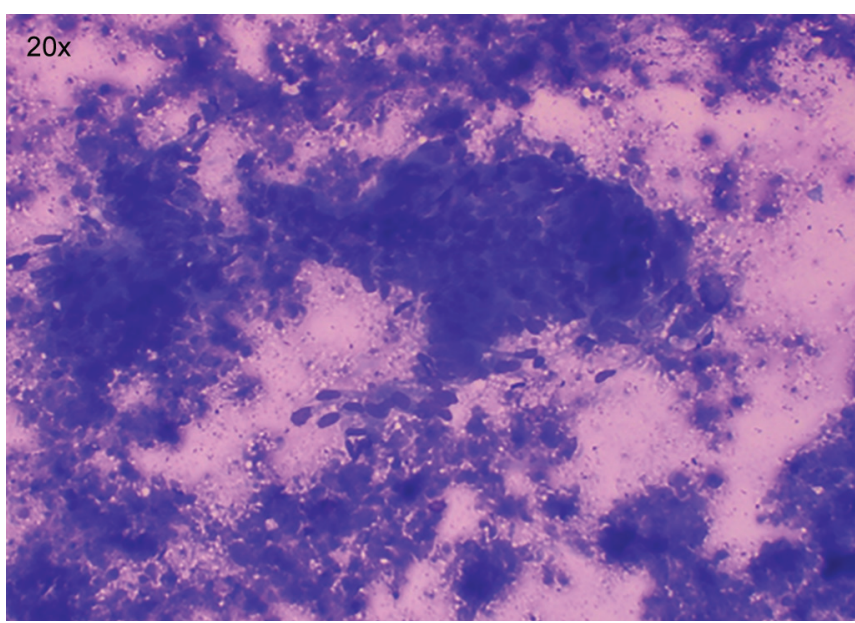

Fig. 2: Individual tumor cells are polygonal and spindle shaped. Evidence of necrosis seen

must represent a distinct clinical entity, and the probability that one is a result of metastatic spread from the other must be reliably excluded. ${ }^{11}$

Among possible combinations, squamous cell carcinoma is by far the most common in lung. Treatment regimens are different for patients with two primaries from those having colorectal cancer with pulmonary metastasis. $^{11}$

For patients with synchronous colorectal and lung cancer, if the condition of the patient permits, synchronous curative resection of the two lesions is the first choice. ${ }^{12}$ After radical resection, according to the pathological type and stage of the tumors, appropriate adjuvant chemotherapy should be given. ${ }^{8,12}$ As far as the literature reports, patients with MPM have a better prognosis than those with a metastasis or recurrent lesion.

Aspergillosis is the most common filamentous fungal infection seen in immunocompromised patients. The significance of this infection is increasing, with growing numbers of patients with malignancy, organ transplantation, steroid abuse, and autoimmune and inflammatory conditions. ${ }^{4}$ Aspergillus fumigatus and Aspergillus flavus are the most common etiological agents. The frequency of $A$. terreus infections varies from 3 to $12.5 \%$. Aspergillus terreus was significantly more likely to be nosocomial in origin. It is resistant to the commonly used drug amphotericin B and hence, treatment of choice is voriconazole or itraconazole. ${ }^{13}$

\section{CONCLUSION}

Thus, we report a rare case of synchronous nonsmall cell lung cancer and rectal cancer with adrenal metastasis and secondary fungal infection with $A$. terreus. As per our knowledge, the occurrence of MPM with secondary fungal infection has not been reported till date. 


\section{REFERENCES}

1. Chaudhuri AD, Bhuniya S, Pandit S, Mukherjee S, Bhanja P, Karmakar R, Dhua A, Saha M. A rare case of concurrent multiple primary lung cancer of different histological types. Indian J Chest Dis Allied Sci 2011 Jul-Sep;53(3):181-183.

2. Liu Z, Liu C, Guo W, Li S, Bai O. Clinical analysis of 152 cases of multiple primary malignant tumors in 15,398 patients with malignant tumors. PLoS ONE 2015 May;10(5):e0125754.

3. Spratt JS Jr, Hoag MG. Incidence of multiple primary cancers per man-year of follow up: 20-year review from the Ellis Fischel State Cancer Hospital. Ann Surg 1966 Nov; 164(5):775-784

4. Kousha M, Tadi R, Soubani AO. Pulmonary aspergillosis: a clinical review. Eur Respir Rev 2011 Sep;20(121):156-174.

5. Zhao J, Tan Y, Wu Y, Zhao W, Wu J, Ji M, Shi L, Jiang J, Wu C. A rare case of eight multiple primary malignant neoplasms in a female patient: a case report and review of the literature. Oncol Lett 2015 Feb;9(2):587-590.

6. Sakellakis M,Peroukides S, Iconomou G, Boumpoucheropoulos S, Kalofonos H. Multiple primary malignancies: a report of two cases. Chin J Cancer Res 2014 Apr;26(2):215-218.
7. Schoenberg BS. Multiple primary malignant neoplasms. The Connecticut experience, 1935-1964. Recent Results Cancer Res 1977;58:1-173.

8. Goya T, Miyazawa N, Kondo H, Tsuchiya R, Naruke T, Suemasu K (1989) Surgical resection of pulmonary metastases from colorectal cancer. 10-year follow-up. Cancer 64:1418-1421

9. Evans HS, Møller H, Robinson D, Lewis CM, Bell CMJ, Hodgson SV. The risk of subsequent primary cancers after colorectal cancer in southeast England. Gut. 2002;50(5): 647-652.

10. Peng YF, Gu J. Synchronous colorectal and lung cancer: report of three cases. World J Gastroenterol 2008 Feb;14(6):969-973.

11. Németh Z, Czigner J, Iván L, Ujpál M, Barabás J, Szabó G. Quadruple cancer, including triple cancers in the head and neck region. Neoplasma 2002 Jun;49(6):412-414.

12. Zhao W, Hu F, Wang J, Jin X, Zhang X, Li H, Li Y, Ren M. Synchronous primary cancer of the rectum and lung: a case report. Chinese-German J Clin Oncol 2012 Jan;11(1):55-58.

13. Steinbach WJ, Benjamin DK Jr, Kontoyiannis DP, Perfect JR, Lutsar I, Marr KA, Lionakis MS, Torres HA, Jafri H, Walsh TJ. Infections due to Aspergillus terreus: a multicenter retrospective analysis of 83 cases. Clin Infect Dis 2004 Jul;39(2):192-198. 